Herz 2018 · 43:101-102

https://doi.org/10.1007/s00059-018-4681-7

(c) Springer Medizin Verlag $\mathrm{GmbH}$, ein Teil von Springer Nature 2018

CrossMark

\section{Thomas Eschenhagen ${ }^{1,2}$}

${ }^{1}$ Institut für Experimentelle Pharmakologie und Toxikologie, Universitätsklinikum Hamburg Eppendorf (UKE), Hamburg, Deutschland

${ }^{2}$ DZHK (Deutsches Zentrum für Herz-Kreislauf-Forschung), Hamburg/Kiel/Lübeck, Deutschland

\title{
Kardiologie 2018
}

\section{Von der Grundlagenforschung zur Hochleistungsmedizin}

Das Motto der diesjährigen Frühjahrstagung der Deutschen Gesellschaft für Kardiologie (DGK) „Kardiologie 2018 von der Grundlagenforschung zur Hochleistungsmedizin“"spiegelt die Bandbreite der modernen Kardiologie wider. Für das Themenheft „Kardiologie 2018“ habe ich einzelne Entwicklungen ausgewählt, die ich wissenschaftlich besonders spannend finde, die Anwendungspotenzial haben und in absehbarer Zeit das Repertoire diagnostischer und therapeutischer Möglichkeiten der Kardiologie erweitern können.

Wenige Bereiche der Kardiologie waren in den letzten Jahrzehnten so erfolgreich wie die Prävention von Herzinfarkten und die Behandlung des akuten Koronarsyndroms. Dennoch bleibt der Herzinfarkt mit seinen Folgen eine der häufigsten Ursachen für Morbidität und Mortalität. Entsprechend stehen neue Ansätze zur Verhinderung von Atherosklerose und Atherothrombose weit oben in den Forschungsstatistiken der letzten Jahre. Erfolgsgeschichten wie die Statine und PCSK9(Proproteinkonvertase Subtilisin/Kexin Typ 9)-Inhibitoren zur pharmakologischen Senkung des LDLCholesterins (LDL: „low-density lipoprotein") stehen neben bislang enttäuschenden Interventionen in den HDLCholesterin Stoffwechsel (HDL: „highdensity lipoprotein“). Eine der großen Fragen im Feld ist, ob die entzündliche Komponente der Atherothrombose als solches ein sinnvoller Ansatzpunkt ist. Dieser Frage widmet sich der Beitrag von Herrn Söhnlein.

Der Verlust von kontraktiler Herzmuskelmasse ist irreversibel und führt zur Herzinsuffizienz, die medikamentös zu verlangsamen, jedoch nicht aufzuhalten oder zu revertieren ist. Die Hoffnung, dieses grundlegende Dilemma der Kardiologie zu durchbrechen, begründet die zum Teil übergroßen Hoffnungen in die regenerative Kardiologie. Nach 15 Jahren intensiver Forschung ist nun nüchtern festzustellen, dass sich Herzmuskelzellen im Laufe des Lebens nur in sehr geringem Umfang regenerieren (ca. 0,5-2\% pro Jahr) und weder Stammzellen aus dem Knochenmark noch aus dem Herzen Anteil daran haben. Stattdessen handelt es sich um Zellteilung von bereits ausgeformten Herzmuskelzellen. Das allerdings eröffnet spannende Ansätze zur pharmakologischen Stimulation der Zellteilung. Und durch enorme Fortschritte in der Herstellung von Herzmuskelzellen aus pluripotenten Stammzellen gewinnt ein anderer Ansatz an Umsetzungspotenzial: die Einbringung von aus Stammzellen hergestellten Herzmuskelzellen in das erkrankte Herz, entweder als Zellsuspension oder als Herzpflaster. Diese Strategie wird im Beitrag von Frau Reinsch und Herrn Weinberger diskutiert.

Das humane Genomprojekt hat, für viele unerwartet, gezeigt, dass nur ein sehr kleiner Anteil der genetischen Information tatsächlich für die etwa 20.000 Proteine des Körpers kodiert (1-3\%). Wofür der gerade bei Homo sapiens große Rest steht, ist weitgehend unklar. Einen wichtigen Anteil davon beginnt die Forschung aber zu verstehen, nämlich ein hoch ausgefeiltes Netzwerk von kleinen, nichtkodierenden Nukleinsäuren (nichtkodierende RNA), die modulierend in die Expression der letztlich funktionsausübenden Proteine eingreifen. Mehrere Argumente machen nichtkodierende RNA zu aussichtsreichen Kandidaten für eine therapeutische Modulation:

1. Genetische Studien lokalisieren einen Großteil aller das kardiovaskuläre Risiko beeinflussenden Genvarianten gerade in solchen regulatorischen Bereichen und nicht in den proteinkodierenden Anteilen.

2. Pharmakologische Modulation von Netzwerken ist zumindest theoretisch besser als Proteine ganz an- oder abschalten.

3. Die RNA lassen sich relativ gut therapeutisch beeinflussen. Tatsächlich sind erste mikro-RNA-basierte Therapien zugelassen.

Einen Überblick über diesen florierenden Forschungsbereich gibt der Beitrag der Autoren Bührke, Bär und Thum.

Kardiologie steht für die Behandlung großer Patientengruppen mit ähnlichen Arzneimitteln („one for all“), z. B. ACEHemmer (ACE: , angiotensin-converting enzyme") und Betablocker bei Hypertonie oder chronischer Herzinsuffizienz, unabhängig von der individuellen Pathogenese der Erkrankung. Der gegenteilige Trend wird in der modernen Onkologie verfolgt, in der Tumoren als Konsequenz spezifischer Mutationen verstanden und individuell behandelt werden (z. B. Philadelphia-positive chronische myeloische Leukämien [CML] und Glivec $^{\circledR}$ ). Dieses Konzept lässt sich in der Kardiologie am ehesten auf die seltenen genetisch determinierten Erkrankungen übertragen. Der Beitrag der Autoren Sedaghat- 
Hamedani, Katus und Meder tut dies am Beispiel der Kardiomyopathien und zeigt Perspektiven eines individualisierten diagnostischen und therapeutischen Konzepts auf, das mittelfristig auch auf häufigere kardiovaskuläre Erkrankungen anwendbar sein wird.

Die Beiträge zeigen, dass sich die kardiovaskuläre Medizin in einer spannenden Entwicklungsphase befindet und großes Potential hat, mit neuen Ansätzen die Behandlung von Patienten grundlegend $\mathrm{zu}$ verbessern. Das ist gerade angesichts der weltweiten Zunahme von Herz-Kreislauf-Erkrankungen dringend notwendig.

Ihr

Prof. Dr. Thomas Eschenhagen

\section{Korrespondenzadresse}

Prof. Dr. T. Eschenhagen

Institut für Experimentelle Pharmakologie und Toxikologie, Universitätsklinikum Hamburg Eppendorf (UKE)

Martinistraße 52, 20246 Hamburg, Deutschland t.eschenhagen@uke.de

Interessenkonflikt. T. Eschenhagen gibt an, dass kein Interessenkonflikt besteht.

\section{Schwaches Herz durch veränderten Stoffwechsel}

Das Herz bezieht seine Energie vorrangig aus Fettsäuren. Stellt sich der Stoffwechsel jedoch auf andere Energiequellen um, kann Herzinsuffizienz die Folge sein. Das unterstreicht nicht nur die Rolle des Stoffwechsels bei Herzinsuffizienz, die Erkenntnisse sind auch für den Einsatz bestimmter Krebsmedikamente von Bedeutung.

Es ist schon länger bekannt, dass Herzinsuffizienz eine Veränderung im Stoffwechsel nach sich zieht. Während gesunde Herzen ihren Energiebedarf zu mehr als $75 \%$ aus Fettsäuren gewinnen, steigen geschwächte Herzen auf Kohlenhydrate als bevorzugte Energiequelle um. Doch der Zusammenhang gilt auch umgekehrt, wie Wissenschaftler aus dem Deutschen Krebsforschungszentrum (DKFZ) und der Universität Heidelberg nun festgestellt haben: Eine Umstellung des Stoffwechsels von Fettsäuren als Energiequelle zugunsten von Kohlenhydraten verursacht Herzinsuffizienz.

Als biochemischen Schlüssel zu diesem Effekt identifizierte das Team ein Rezeptormolekül namens Notch-1. Mit Hilfe eines therapeutischen Antikörpers, der vorwiegend in Zellen der Blutgefäßwand wirkt, hatten die Wissenschaftler bei Mäusen den Notch-1-Signalweg blockiert. In der Folge entwickelten die Tiere eine Herzinsuffizienz. In einem weiteren Experiment nutzten die Forscher einen genetischen Trick und züchteten Mäuse, bei denen sich die Notch-1-Signalübertragung gezielt in den Endothelzellen, die die Blutgefäße von innen auskleiden, ausschalten lässt. Wieder erkrankten die Tiere innerhalb weniger Wochen an Herzinsuffizienz.

Damit Nährstoffe aus den Blutgefäßen in den Herzmuskel gelangen, müssen sie durch die Endothelzellen transportiert werden. Wird Notch-1 blockiert, stehen dem Herzen weniger Fettsäuren zur Verfügung und es muss auf Zucker als Energiequelle umsteigen. Dabei wird ein weiterer Signalweg namens mTORaktiviert, der zu einem Wachstum des Herzmuskels und schließlich zur Herzinsuffizienz führt. Ein Blockieren dieses Signalwegs konnte im Experiment verhindern, dass die Mäuse eine Herzinsuffizienz entwickelten. Denselben Effekt hatte eine extrem kohlenhydratarme Diät.

Die Ergebnisse zeigen, welche wichtige Bedeutung eine Veränderung des Stoffwechsels, etwa aufgrund von angeborenen oder erworbenen Stoffwechselerkrankungen, für den Verlauf einer Herzinsuffizienz hat. Ferner haben sie darüber hinaus eine weitreichende Bedeutung für die Krebsforschung: Notch1 erfüllt im Körper vielfältige Funktionen und stellt ein Onkogen dar. Mutationen, die zu einer verstärkten Aktivierung führen, können das Entstehen und die Entwicklung von Tumoren begünstigen.

Das macht den Notch-Signalweg zu einem interessanten Angriffspunkt für die Krebstherapie - zumindest theoretisch. Denn bei einigen frühen klinischen Studien mit einem Wirkstoff, der diesen Signalweg blockiert, erkrankten Probanden an einer Herzinsuffizienz. Die Ergebnisse der aktuellen Studie können diese vermeintlich überraschende $\mathrm{Ne}$ benwirkung nun erklären. Möglicherweise könnten spezielle Diäten oder eine zusätzliche Blockade des mTOR-Signalwegs während der Behandlung helfen, die lebensbedrohliche Nebenwirkung Herzinsuffizienz zu verhindern.

Literatur: Jabs M, Rose AJ, Lehmann LH et al (2018) Inhibition of endothelial Notch signaling impairs fatty acid transport and leads to metabolic and vascular remodeling of the adult heart. Circulation

DOI 10.1161/CIRCULATIONAHA.117.029733

Quelle: DKFZ, Heidelberg 